\title{
In-ear medical devices for acoustic therapies in tinnitus treatments, state of the art
}

\author{
David Ibarra ${ }^{a, b, *}$, Francisco Tavira-Sanchez ${ }^{c}$, Manuel Recuero-Lopez , \\ Brian W. Anthony ${ }^{b}$ \\ ${ }^{a}$ Tecnológico de Monterrey, Escuela de Ingeniería y Ciencias, Campus Mexico City, Calle del Puente 222, Ejidos de Huipulco, Tlalpan, \\ 14380 Mexico D.F., Mexico \\ ${ }^{\mathrm{b}}$ Laboratory for Manufacturing and Productivity, Massachusetts Institute of Technology, 77 Massachusetts Avenue, Cambridge, MA 02139, \\ USA \\ ${ }^{\mathrm{C}}$ Grupo de Investigación en Instrumentación y Acústica Aplicada (I2A2), Universidad Politécnica de Madrid, Carretera de Valencia km. 7, \\ 28031 Madrid, Spain
}

\begin{abstract}
A B S T R A C T
Cochrane reviews indicate there is very limited support for all forms of sound therapy and cognitive behavioral therapy has the strongest support. American Academy of Otolaryngology (AAO) recently published some guidelines which recommends Cognitive Behavioral Therapy (CBT) for tinnitus intervention, and only indicates that sound therapy should be considered an "option" for intervention.

Nevertheless, acoustic therapy could lead to cause changes in the tinnitus perception and has been appreciated by the affected people for years. In the last decades, the use of sound or sound enrichment has become a central part of many tinnitus management programs used by audiologists, whether the intention was to mask tinnitus, suppress tinnitus, or interrupt the tinnitus generating neural activity.

Several acoustic therapies have been developed and implemented in the last 40 years, but how can we determine which one is the most effective? We can determine the effects based on the results reported in many research studies, but in those studies are many factors that differ from one study to another, like in-ear medical devices used to apply acoustic therapy for tinnitus treatment.

In this article, we review and analyze the different types of in-ear medical devices used in the most recently acoustic therapies in treatments against tinnitus, allowing us to identify the pros and cons. By our analysis, an optimal medical device could be characterized to enhance the application of acoustic therapies and in consequence the global results of the sound therapies that already exist. In this review, it was considered acoustic therapies, the technology implemented in medical devices and the clinical needs.
\end{abstract}

Keywords:

Medical device

Hearing aid

Acoustic therapy

Tinnitus

\section{Introduction}

Tinnitus is known to affect between $5 \%$ and $15 \%$ of the population [1]. About half of the people with tinnitus are concerned about it, and it is reported that about $2 \%$ indicate that

\footnotetext{
* Corresponding author.

E mail address: david.ibarra@itesm.mx (D. Ibarra).
}

tinnitus significantly affects their life. Tinnitus may have a direct effect on a person's emotional wellbeing, their hearing, and ability to sleep and to concentrate. These in turn impact basic life functions such as socialization and relaxation [2]. In severe cases, it can interfere with the individual's ability to perform adequately on the job, or contribute to psychological disorders such as depression, suicide ideation, posttraumatic stress disorder, anxiety, and anger [3]. 
The Committee on Hearing, Bioacoustics, and Biomechanics (CHABA) defined tinnitus as "the conscious experience of a sound that originates from the patient's own head." It can be distinguished between objective tinnitus (somatosounds) due to peripheral vascular abnormalities, among others, detected by stereoscopic inspection, and subjective tinnitus that cannot be heard by others [4]. From an acoustic point of view tinnitus is characterized by its intensity and its frequency content [5].

Generally speaking, sound therapy is the appropriate and conscious use of sound energy to promote well-being in humans and also the expansion of consciousness. The therapy through sounds is based on the knowledge that all matter is vibrating at specific frequencies. These therapies can influence physiological, cognitive and even psychological variables [6].

Many different acoustic therapies for Tinnitus approaches have been marketed over the last 40 years though with varying success. Some of the most widely used therapy approaches for which positive results have been reported include tinnitus masking (wearing an ear level masking device), Tinnitus Retraining Therapy (which includes counseling and sound therapy principles), and Neuromonics (a music-based approach combined with counseling) [7]. However, no single treatment is $100 \%$ effective. Shortly afterwards, we will explain these therapies and their sound generators more in detailed.

Audiologists have always known that sounds could interfere with tinnitus [8 11], though there is no consensus on how to measure the effectiveness of the outcomes of tinnitus treatments so that they remain inconclusive [12 14].

Beck explains that some medical devices are effective in assisting people with tinnitus by helping the brain to distinguish between true sounds and pseudo sound (tinnitus), increasing neural activity and assisting the brain in correcting for the potential negative effects of disinhibition, partial masking of tinnitus, and improving the ability to cope with tinnitus by reducing the stress in their lives [15]. In the Audiological Tinnitus Management (ATM) protocol, Henry et al. [16] recommended the use of a combination of instruments (amplification and noise generator in the same device) for a blended benefit of amplification and constant broadband noise to achieve this enhanced soundscape.

\section{In-ear medical devices}

Hearing aids were programmed to assist hearing instead of a focus on tinnitus. They have become common therapeutic tools in the audiological management of tinnitus [17]. However, there is currently no evidence to support or refute the provision of hearing aids as a primary intervention in the management of tinnitus in patients with co-existing hearing loss [18]. The lack of information about the head causes directivity reduction and distortions. The signals that arrive at the array are distorted by the so-called head shadow effect, which must be considered in the design of hearing aids [19].

It is only fairly recently that the potential physiological origins of tinnitus [20] and mechanisms of sound-based treatments have become clearer [21,22]. Moreover, some of the patients suffer from psychiatric comorbidities as depression or anxiety.
Nevertheless, hearing aids have been a popular choice for tinnitus intervention, Kochkin and Tayler [17] did a survey study of 230 hearing care professionals suggests that six out of 10 patients $(60 \%)$ experienced minor to major relief of tinnitus when wearing hearing aids, and a total of one in five (22\%) receive major relief. Less than $2 \%$ of patients experience a worsening of their tinnitus when wearing hearing aids, while $39 \%$ receive no benefit. Due to the most effective settings of hearing aid for tinnitus suppression may not be same as for enhancing communication [23]. For example, hearing aids can lead to habituation of tinnitus for patients with tinnitus and hearing loss but not with hyperacusis [24].

Most of the devices that exist in the commercial market for tinnitus treatment are hearing aids, which have a masking signal implemented in the device for the therapy in the majority of the cases [25]. In the case of hearing aids used in tinnitus treatments should be open ear hearing aids [26], because with a partial occlusion is possible to avoid an increase in the perception of the tinnitus instead of a complete occlusion in the ear canal with other kind of hearing aids.

Other type of implemented technology is the cochlear implantation, which allows the preservation of residual hearing after cochlear implantation and the synergistic use of electric and acoustic stimulation (EAS). EAS is a well-accepted therapeutic treatment for subjects with profound hearing loss in the higher frequencies and no or mild hearing loss in the low frequencies. Several manufacturers offer individual soft electrodes specially designed for hearing preservation as well as combined electric-acoustic audio processors for tinnitus treatment [27].

\subsection{Types of in-ear medical devices}

Any device that amplifies sound might be helpful for tinnitus management. There are numerous articles underlining this effect, and even the AAO guidelines support the use of hearing aid for tinnitus management. Most in-ear medical devices for tinnitus treatment are hearing aids that are classified depending of the characteristics of the treatment and the needs of patient [28]. The classification of different types of in-ear medical devices in Tinnitus Management is showed in Fig. 1.

Behind-the-ear (BTE) medical devices consist of a hard plastic case worn behind the ear and connected to a plastic ear mold that fits inside the outer ear. The electronic parts are held in the case behind the ear. Sound travels via the hearing aid through the ear mold and into the ear. In a study performed by Munhoes Dos Santos et al. [29], 66\% of the patients prefer BTE hearing aids with open molds than the other kind of molds.

In-the-ear (ITE) medical device fits completely inside the outer. The case holding the electronic components is made of hard plastic. Some ITE devices may have certain added features installed, such as a telecoil, which is a small magnetic coil that allows users to receive sound through the circuitry of the hearing aid, rather than through its microphone.

In the canal (ITC) medical device is made to fit the size and shape of a patient's ear canal. A completely in canal (CIC) medical device is nearly hidden in the ear canal. Because they are small, canal aids may be difficult for a patient to adjust and 


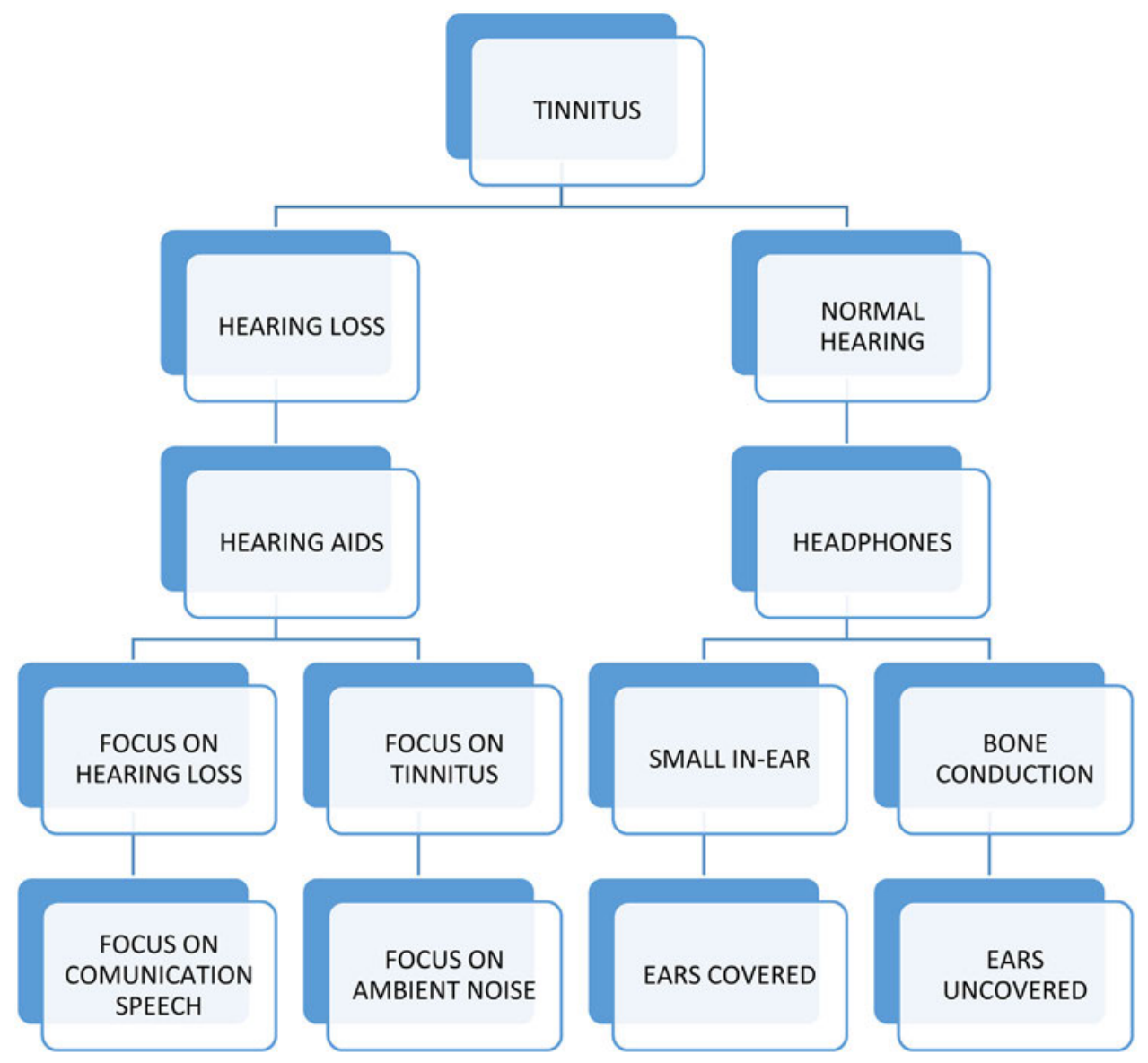

Fig. 1. Different types of devices in tinnitus management.

remove [30]. In addition, canal aids have less space available for batteries and additional devices, such as a telecoil.

Personal In Ears (PIE) is a Full-Shell style device partiallyto-fully fill the outer ear. They are custom manufactured to fit securely, and be as aesthetically pleasing as possible. All components reside within a lightweight plastic shell, which is simple to insert and remove. These devices are used widely in audio applications as monitors for the musicians.

Invisible In The Canal (IIC) is a style of hearing aids that fits inside the ear canal completely, what makes it out of view even when looking directly into the ear bowl. A comfortable fit is achieved because the shell of the aid is custom-made to the individual ear canal and the sound can be collected more naturally by the shape of the ear. Some models are controlled by a mobile phone to alter memory and volume settings.

Middle Ear Implants (MEI) help increase the transmission of sound to the inner ear. MEIs are small implantable devices that are typically attached to one of the tiny bones in the middle ear. When they receive sound waves, MEIs vibrate and directly move the middle ear bones. This creates sound vibrations in the inner ear, which helps to detect the sound. This device is generally used by people who do not benefit from or are unable to use traditional hearing aids, such as obstruction of the outer or middle ear, who cannot wear hearing aids and earmoulds, or who are unable to benefit from sound amplification. There is variance in authorized indications of middle ear implants across countries: conductive, sensorineural and mixed hearing loss. In the following table is shown some MEI examples and what certifications have obtained (Table 1) [31 34].

Cochlear Implant (CI) is an implanted electronic device that can produce useful hearing sensation by electrically stimulating nerves inside the inner ear. Cochlear implants currently consist of two main components: an external component comprised of an externally worn microphone, sound processor and transmitter system, and an internal component, comprised of an implanted receiver and electrode system, which contains the electronic circuits that receive signals from the external system and send electrical signals to the inner ear.

Digital circuits convert sound waves into numerical codes, similar to the binary code of a computer, before amplifying them. Because the code also includes information about a sound's pitch or loudness, the system can be specially programmed to amplify some frequencies more than others do. In addition, Digital circuitry, i.e., can be used in all types of medical devices and its design requires a set of filters that provides reasonable audiogram matching for the concerned type of hearing loss [35]. Some sound processors were developed to implement an automatic scene-classifying algorithm, which is an improvement over the previous generation of sound processing algorithms and is suitable for use by traditional cochlear implant and Hybrid L24 implant recipients [36].

Other kinds of devices are the tinnitus maskers that increase the level of sound in the listener's environment and therefore 
Table 1

A summary of six middle ear implant systems.

\begin{tabular}{|c|c|c|c|c|}
\hline Manufacturer & Status & Type & Location of microphone & Location of transducer \\
\hline Implex & CE mark* & Piezo & Ear canal wall & Head of stapes \\
\hline Otologics LLC & FDA trial and CE mark & $\mathrm{EMe}$ & Button BTE & Probe in incus \\
\hline Soundtec & FDA approval and Cdn. approval & EM & In the ear shell & Incudo stapedial \\
\hline Symphonix & FDA approval and CE mark & EMe & Button BTE & Incudo stapedial \\
\hline Envoy Medical Co. & FDA approval and CE mark & Piezo & & Head of stapes \\
\hline MedEl & FDA approval and CE mark & EM & Button BTE & Incudo stapedial \\
\hline
\end{tabular}

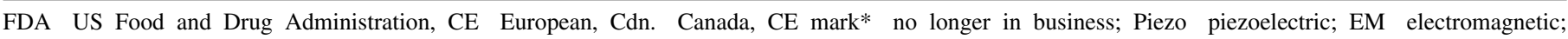
EMe Electromechanical.

mask the ringing in the sufferer's head with a calming, less intrusive sound. It has been estimated that up to $90 \%$ of patients with tinnitus may benefit from amplification [37,38]. Even for patients who are marginal hearing aid candidates, highfrequency amplification (i.e., primary gain at $34 \mathrm{kHz}$ ) may be readily accepted and beneficial.

\subsection{In-ear medical devices as instrument for current acoustic therapies}

In 2012, Attanasio et al. [39] suggested that Mozart therapy could be a valid alternative to the common sound therapy methods with tinnitus patients. The study was done to evaluate the presence of the Mozart effect as indexed by a variation in tinnitus intensity and tolerability but there is no clear proof that Mozart is more beneficial for tinnitus management than any other music.

Musical stimuli may provide a different, and in some cases, superior option for sound therapies. It may be preferable for some people but not for others. The use of amplification, combined with flexible music-like signals containing relaxation inducing properties may be a further asset to tinnitus management procedures [2]. An example is the use of fractal (randomly generated) tones, which differs from conventional music in terms of its familiarity but similar preferences for using music for relaxation and tinnitus sound therapy appear to apply. Examples are Bach's Air in G, Albinoni's Adagio, and two of Vivaldi's Four Seasons.

The use of sound-generating devices specifically for treating tinnitus developed with the advent of hearing aids as portable electronics became available in the 1950s. Sound generating devices have been used formally as part of structured tinnitus treatment programs, and not simply as maskers, along 30 years. Some related examples are as follows:

Tinnitus retraining therapy (TRT), which was first proposed by Jastreboff [40], is a method for treating tinnitus and decreased sound tolerance. TRT counseling is based on the neurophysiological model of tinnitus, and sound therapy, which is performed with or without instrumentation $[41,42]$. The aim of sound therapy is to decrease the contrast between tinnitus and background neuronal activity. The primary purpose is to mitigate the tinnitus with improved audibility considered a secondary benefit. While the masking method uses sound to achieve immediate relief, TRT uses sound enrichment to promote long-term habituation to tinnitus.
Tyler [43] at the University of Iowa developed the Tinnitus Activities Treatment (TAT), which takes a different approach. It looks at four specific functions that can be impaired because of tinnitus. Those are identified as thoughts and emotions, hearing, sleep, and concentration. Sound therapy is used to help decrease the prominence of the tinnitus and help ease the patient into habituation.

Neuro-music therapy according to the Heidelberg Model for chronic tinnitus strives for an integration of strategies to manage the psychological state and possibly restore the assumed underlying neurophysiological reorganization in both the lemniscal and extralemniscal auditory pathways [44]. Results from several clinical trials [45 47] demonstrate that this integrative music therapy approach could reduce tinnitus distress $(80 \%$ as measured by the Tinnitus Questionnaire).

\section{Technological innovations in commercial devices}

While the market has produced a number of good options for a combination hearing aid and tinnitus therapy devices, the research has not been conducted to evaluate whether these instruments provide greater benefit to patients than the use of hearing aids alone [48].

All commercialized sound therapy devices are more suitable for certain patients, studies have only been reported by people affiliated with the company, so the method has no real credibility. Nevertheless, some applicable facts are as follows:

Neuromodulation uses sound stimulation for tinnitus aims to disrupt aberrant neural firing. The sound-stimulation regime is personalized to each individual patient and is a sequence of tones carefully selected to span the frequency corresponding to the dominant tinnitus pitch $[49,50]$. Tass, in his Rebuttal to reply the G. Rucker and G. Antes criticism [51], explains that the results demonstrate that acoustic CR neuromodulation has an effect on the postulated pathophysiological causes of tinnitus. In contrast, common therapies such as TRT or CBT, aim at one of three response systems (behaviour, cognition and physiological reactivity) and are unlikely to have an effect on the pathophysiological causes of tinnitus.

For Neuromonics, the sound-based component of the treatment promotes the desensitization to patients' tinnitus perception using sound stimuli. Its more acceptable to patients than broadband masking noises [52,53]. In this case, there is a subjective evaluation with TRQ (Tinnitus Reaction 
Questionnaire) and specific neurophysiological effects have been explored [54].

Widex Zen plays random, fractal tones with harmonic, but not predictable, relationships that can be used for relaxation or for making tinnitus less noticeable [55]. There is a range of different Zen programs and each sound type can be adjusted to individual preference in terms of pitch, time and gain. The device is recommended to be part of a more holistic audiological management program incorporating education and counseling [56,7]. The investigation is based on the hypothesis that bothersome tinnitus involves limbic system activity where tinnitus is associated with a stress response, and that music has de-stressing properties. This therapy has TRQ scores as a result of its efficacy.

\subsection{Application of electric stimulation}

Serenade is delivered as a part of a broader tinnitus treatment program. The suppression acts where the patterned sounds modulate auditory cortical activity and interrupt tinnitus generation. The stimuli is charge-balanced, bi-phasic pulses and delivered to the subject at various loudness levels using monopolar mode (one electrode located in the cochlea and the other located extra cochlear, deep to the temporalis muscle). Stimulus rate ranges from 10 to $5000 \mathrm{~Hz}$ and stimulus duration is $360 \mathrm{~s}$ [57]. There are a few case studies about this therapy that demonstrate specific neurophysiological effects on auditory cortical activity [58].

\section{Clinical need in the implement of in-ear medical devices in acoustic therapies}

There are some comparisons between the uses of diverse inear medical devices, as a hearing aids, sound generators or simple CD players with headphones, being the case of Parazzini et al. [59] that compared the use of sound generators and openear hearing aids in tinnitus retaining therapy. Although the absence of important hearing loss in the speech range, identical results were obtained for both sound generators and hearing aids. Some studies carried by Folmer and Caroll [60] demonstrate that the acoustic therapies carry out with headphones and CD players have better results than those provided in hearing aids, especially in high frequency range. Munhoes dos Santos et al. [29] considered the efficacy of different ear mold styles in tinnitus intervention and found that the benefit offered by sound generators were independent of the type of ear molds. Otherwise Hobson et al. [61] determined that in this case hearing aids were not effective for tinnitus management. They reported that hearing aids had a positive impact on hearing handicap. Nevertheless, it was difficult to support the use of hearing aids for reducing tinnitus handicap.

According to the study of data mining of the records of the audiology patient, there are factors influencing the choice of the type of hearing aid. Patients with tinnitus tended more to wear ITE hearing aids [62]. The relationship between the wearing of a tinnitus masker and hearing aid type, among patients diagnosed with tinnitus, had the overall chi-squared value [63]. When the tinnitus spectrum contains frequencies higher than $6 \mathrm{kHz}$, the tinnitus cannot be masked with Tinnitus Control Instruments or Hearing Aids because of the limitations imposed by the frequency characteristics of these instruments $[16,64]$. Therefore, for patients with tinnitus of higher frequencies, Portable Music Player should be effective with output sounds with frequencies greater than $10 \mathrm{kHz}$.

On the other hand, in the most of the cases, patients remove their hearing aids before going to bed, and it is often reported that 'trouble sleeping' is the most common complaint of people with tinnitus [65].

Table 2

Tinnitus issues vs solutions.

\begin{tabular}{|c|c|}
\hline Issues & Solutions \\
\hline Lack of knowledge about tinnitus & Awareness about tinnitus, there is not cure, but it can minimized \\
\hline $\begin{array}{l}\text { Most of medical devices in the commercial market for } \\
\text { tinnitus are hearing aids }\end{array}$ & Proportionate more devices specialized for tinnitus treatment \\
\hline $\begin{array}{l}\text { The measure outcomes are imprecise and not realistic, } \\
\text { just subjective point of view }\end{array}$ & Objective evaluations as FMRI, EEG, Brain mapping, Neuroimaging, etc. \\
\hline $\begin{array}{l}\text { Hearing aids are programmed to assist hearing loss instead } \\
\text { of a focus on tinnitus }\end{array}$ & Propose new devices just for patients with tinnitus, reinforcing the high frequencies \\
\hline $\begin{array}{l}\text { The majority of the performed studies were made with } \\
\text { heterogeneous samples of patients }\end{array}$ & $\begin{array}{l}\text { The samples of the patients have to be more homogenous in order to do a complete } \\
\text { study }\end{array}$ \\
\hline $\begin{array}{l}\text { There are not specific neurophysiological effects and the } \\
\text { commercial devices are focused on the patients' relaxation }\end{array}$ & Standardization and the same regulations for the commercial devices. \\
\hline $\begin{array}{l}\text { There are not the characteristics of background sounds which } \\
\text { interfere with speech communication and the annoyed sounds }\end{array}$ & $\begin{array}{l}\text { A special device with the characteristics of the patients for some determined sounds. } \\
\text { Use new technology in digital hearing aids reduce the background noise }\end{array}$ \\
\hline The tinnitus population is increasing in the last years & Awareness about personal stereo devices for adequate use with moderate volume \\
\hline
\end{tabular}


In the tinnitus assessment, there are few Randomized Controlled Trials (RCT). The paucity of RCTs for medical device effects on tinnitus may be due to the high cost of such trials (e.g., cost per device). The tinnitus benefits for those devices are secondary and there may not be the same regulations or commercial drivers as for standardized clinical trials. That is the reason why in some studies have been used various assessment tools that have questionable validity [66].

The unilateral cochlear implant may improve tinnitus in patients, a significant percentage of cases is demonstrated [67]. Studies of cost-benefit for evaluating tinnitus as an indication of cochlear implant should be performed. With the intention of replace the hearing aids.

Several clinicians agree that a combination of tinnitus management strategies is more effective than one form of remediation used in isolation, but it

Ls more time consuming. Teaching patients how to use external sounds reduces their perception of chronic tinnitus [60].

For the vast majority of the people in the world, there is a lack of accessibility and affordability to have an in-ear medical device, either for hearing loss or for tinnitus [68]. Designing and producing a brand new low-cost medical device (hearing aid or sound generator) could be the most effective option. For example, the WHO has taken a big step forward in extending its activities to the economic context of medical and assistive technology and focusing more on rehabilitation and community living [69] and the development of an alternative digital hearing suitable for low-resource countries [70].

\subsection{Tinnitus treatment difficulties to use acoustic therapies for in-ear medical devices}

In the following table is explained the difficulties of establishing evidence for tinnitus treatments, a compilation of issues from the bibliography and some possible solutions identified to solve them (Table 2).

\section{Conclusions}

In the majority of the studies, in-ear medical devices are used as hearing aids for assisting hearing rather than tinnitus. For most of them, the devices may not be set optimally for amplification of quiet environmental sounds that are thought to be important for reducing tinnitus audibility. More research is still needed for developing special standardized methodologies targeting tinnitus suppression, as more objective procedures for diagnosing, evaluation and evolution of this kind of disease.

Having reviewed all the relevant literature to that topic, we find that the best optimal in-ear medical device for tinnitus treatment is the bone conduction headphones for patients with no-hearing loss, and in the case of patients with hearing loss, the hearing aid behind the ear with no occlusion.

Despite the commercial sound generators are being a step forward than the research instrument, companies do not give some details about their in-ear device and how is it working and its technological components.

We believe that there are possibilities to increment the good results in acoustic therapies because there is insufficient rigorous neurophysiological evidence either too strongly support outcomes. We need to design and standardize an in-ear medical device for each disease taking into consideration the clinical needs and difficulties identified.

\section{Acknowledgments}

This research has been partially funded by the Tec de Monterrey and MIT Nanotechnology Program.

\section{References}

[1] Heller AJ. Classification and epidemiology of tinnitus. Otolaryngol Clinics North Am 2003;36(2):239 48.

[2] Sweetow R. The use of fractal tones in tinnitus patient management. Noise Health 2013;15(63):96.

[3] Noble W, Tyler R. Physiology and phenomenology of tinnitus: impli cations for treatment. Int J Audiol 2007;46(10):569 74 .

[4] Lenhardt M. Tinnitus devices. In: Encyclopedia of biomaterials and biomedical engineering. 2nd Edition, 2008;p. 2610 9. Four Volume Set.

[5] Kaltenbach JA. The dorsal cochlear nucleus as a participant in the auditory, attentional and emotional components of tinnitus. Hearing Res 2006;216 7. 22434.

[6] Koelsch S. A neuroscientific perspective on music therapy. Ann New York Acad Sci 2009;1169(1):374 84.

[7] Sweetow RW, Sabes JH. Effects of acoustical stimuli delivered through hearing aids on tinnitus. J Am Acad Audiol 2010;21(January (7)):461 73.

[8] Saltzman M, Ersner MS. A hearing aid for the relief of tinnitus aurium. Laryngoscope 1947;LVII(5):358.

[9] Surr RK, Montgomery AA, Mueller HG. Effect of amplification on tinnitus among new hearing aid users. Ear Hearing 1985;6(2):71 5.

[10] Surr RK, Walden BE, Cord MT, Olson L. Influence of environmental factors on hearing aid microphone preference. J Am Acad Audiol 2002;13(6):308 22.

[11] Melin L, Scott B, Lindberg P, Lyttkens L. Hearing aids and tinnitus an experimental group study. Br J Audiol 1987;21(2):91 7.

[12] Meikle MB, Stewart BJ, Griest SE, Henry JA. Tinnitus outcomes assessment. Trends Amplif 2008;12(3):223 35

[13] Kamalski DM, Hoekstra CE, Zanten BGV, Grolman W, Rovers MM. Measuring disease specific health related quality of life to evaluate treatment outcomes in tinnitus patients: a systematic review. Otolar yngol Head Neck Surg 2010;143(2):181 5.

[14] Hoare DJ, Gander PE, Collins L, Smith S, Hall DA. Management of tinnitus in English NHS audiology departments: an evaluation of current practice. J Evaluat Clin Pract 2010;18(2):326 34.

[15] Beck DL. Hearing aid amplification and tinnitus: 2011 overview. Hearing J 2011;64(6):12 4.

[16] Henry J, Schechter M, Zaugg T, Griest S, Jastreboff P, Vernon J, et al. Clinical trial to compare tinnitus masking and tinnitus retraining therapy. Acta Oto Laryngol 2006;126(Suppl. 556):64 9.

[17] Kochkin S, Tyler R. Tinnitus treatment and the effectiveness of hearing aids: hearing care professional perceptions. Hearing Rev 2008;15 (13):14 8 ,

[18] Hoare DJ, Edmondson Jones M, Sereda M, Akeroyd MA, Hall D. Amplification with hearing aids for patients with tinnitus and co existing hearing loss. Cochrane Database System Rev 2014;31(Jan (1)):CD010151.

[19] Ayllón D, Gil Pita R, Rosa Zurera M. Design of microphone arrays for hearing aids optimized to unknown subjects. Signal Process 2013;93 (11):3239 50 . 
[20] Eggermont JJ, Roberts LE. The neuroscience of tinnitus. Trends Neurosci 2004;27(11):676 82.

[21] Norena AJ. Enriched acoustic environment after noise trauma reduces hearing loss and prevents cortical map reorganization. J Neurosci 2005;25(3):699 705.

[22] Schaette R, Kempter R. Development of tinnitus related neuronal hyperactivity through homeostatic plasticity after hearing loss: a computational model. Eur J Neurosci 2006;23(11):3124 38.

[23] Searchfield GD. Hearing aids and tinnitus. New York: Thieme Medical Publishers; 2006. p. 16175.

[24] Gold SL. Clinical management of tinnitus and hyperacusis, 8. The ASHA Leader; 2003. p. 425.

[25] Vernon JA, Meikle MB. Masking devices and alprazolam treatment for tinnitus. Otolaryngol Clinics North Am 2003;36(2):307 20.

[26] Bo LD, Ambrosetti U. Hearing aids for the treatment of tinnitus. In: Tinnitus: pathophysiology and treatment progress in brain research. 2007;p. 3415.

[27] Heyning PVD, Vermeire K, Diebl M, Nopp P, Anderson I, Ridder DD. Incapacitating unilateral tinnitus in single sided deafness treated by cochlear implantation. Ann Otol Rhinol Laryngol 2008;117(9): 64552.

[28] Walden BE, Surr RK, Cord MT, Edwards B, Olson L. Comparison of benefits provided by different hearing aid technologies. J Am Acad Audiol 2000;11(10):540 60.

[29] Ferrari GMDS, Sanchez TG, Pedalini MEB. The efficacy of open molds in controlling tinnitus. Braz J Otorhinolaryngol 2007;73(3): 3707.

[30] Ricketts T, Henry P, Gnewikow D. Full time directional versus user selectable microphone modes in hearing aids. Ear Hearing 2003;24 (5):424 39.

[31] Beutner D, Hüttenbrink KB. Passive and active middle ear implants. GMS Curr Top Otorhinolaryngol Head Neck Surg 2009;8.

[32] Chasin M. Bone anchored and middle ear implant hearing aids. Trends Amplif 2002;6(January (2)):33 8.

[33] Channer GA, Eshraghi AA, Zhong LX. Middle ear implants: historical and futuristic perspective. J Otol 2011;6(2):10 8.

[34] Haynes DS, Young JA, Wanna GB, Glasscock ME. Middle ear implantable hearing devices: an overview. Trends in Amplif 2009;13(January (3)):206 14.

[35] Haridas N, Elias E. Efficient variable bandwidth filters for digital hearing aid using Farrow structure. J Adv Res 2016;7(2):255 62.

[36] Gilden J, Lewis K, Grant G, Crosson J. Improved hearing in noise using new signal processing algorithms with the Cochlear ${ }^{\mathrm{TM}}$ Nucleus ${ }^{\mathbb{R}}$ 6 sound processor. J Otol 2015;10(2):51 6 .

[37] Johnson RM. The masking of tinnitus. In: Tinnitus treatment and relief. Needham Height, MA: Allyn \& Bacon; 1998. p. 16486.

[38] Schechter MA, Henry JA, Zaugg T, Fausti SA. Selection of ear level devices for two different methods of tinnitus treatment. In: VIIth International Tinnitus Seminar Proceedings; 2002.

[39] Attanasio G, Cartocci G, Covelli E, Ambrosetti E, Martinelli V, Zaccone M, et al. The Mozart effect in patients suffering from tinnitus. Acta Oto Laryngologica 2012;132(11):1172 7.

[40] Jastreboff PJ, Hazell JWP. Treatment of tinnitus based on a neuro physiological model. Tinnitus treatment and relief. Needham Height, MA: Allyn \& Bacon; 1998. p. 20117.

[41] Jastreboff PJ, Jastreboff MM. Tinnitus Retraining Therapy (TRT) as a method for treatment of tinnitus and hyperacusis patients. J Am Acad Audiol 2000;11:162 77.

[42] Newman C, Sandridge SA. A comparison of benefit and economic value between two sound therapy tinnitus management options. J Am Acad Audiol 2012;23(2):126 38.

[43] Tyler RS. Tinnitus activities treatment. In: Tinnitus treatment: clinical protocols. New York: Thieme; 2006. p. 11631.

[44] Moller AR. Neural plasticity in tinnitus. Prog Brain Res Reprog Brain 2006;365 72 .

[45] Argstatter H, Krick C, Bolay HV. Musiktherapie bei chronisch tonalem Tinnitus: Heidelberger Modell evidenzbasierter Musiktherapie, 56. Springer Medizin Verlag; 2008. p. 67885.
[46] Argstatter H, Krick C, Plinkert P, Bolay H. Musiktherapie bei nichtto nalem Tinnitus (Tinnitusrauschen). HNO 2010;58(January (11)): 108593.

[47] Nickel AK. Outcome research in music therapy: a step on the long road to an evidence based treatment. Ann New York Acad Sci 2005;1060 (January (1)):283 93.

[48] Henry JA, Frederick M, Sell S, Griest S, Abrams H. Validation of a novel combination hearing aid and tinnitus therapy device. Ear Hearing 2015;36(1):42 52.

[49] Tass PA, Adamchic I, Freund HJ, von Stackelberg T, Hauptmann C. Counteracting tinnitus by acoustic coordinated reset neuromodulation. Restor Neurol Neurosci 2012;30(2):137 59.

[50] Mantini D, Perrucci MG, Gratta CD, Romani GL, Corbetta M. Electrophysiological signatures of resting state networks in the human brain. Proc Natl Acad Sci 2007;104(January (32)):13170 75.

[51] Tass PA, Adamchic I, Freund HJ, von Stackelberg T, Hauptmann C. Rebuttal to reply by G. Rücker and G. Antes on Tass et al. "Counter acting tinnitus by acoustic coordinated reset neuromodulation". Restor Neurol Neurosci 2012;30(2):235 7.

[52] Davis PB, Paki B, Hanley PJ. Neuromonics tinnitus treatment: third clinical trial. Ear Hearing 2007;28(2):242 59.

[53] Davis PB, Wilde RA, Steed LG, Hanley PJ. Treatment of tinnitus with a customized acoustic neural stimulus: a controlled clinical study. Ear Nose Throat J 2008;87(6):330 9.

[54] Goddard JC, Berliner K, Luxford WM. Recent experience with the neuromonics tinnitus treatment. Int Tinnitus J 2009;15(2):168 73.

[55] Kuk F, Peeters H, Lau CL. The efficacy of fractal music employed in hearing aids for tinnitus management. Hearing Rev 2010;17(10): 3242.

[56] Jeppesen AMK, Andersen HP. Tinnitus and the Widex Zen program. Widexpress 2011;(February (27))

[57] Reavis KM, Chang JE, Zeng F G. Patterned sound therapy for the treatment of tinnitus. Hearing J 2010;63(11):21 2.

[58] Zeng F G, Tang Q, Dimitrijevic A, Starr A, Larky J, Blevins NH. Hearing research. Hearing Res 2011;207:61 6.

[59] Parazzini M, Bo LD, Jastreboff M, Tognola G, Ravazzani P. Open ear hearing aids in tinnitus therapy: an efficacy comparison with sound generators. Int J Audiol 2011;50(8):548 53.

[60] Folmer R, Carroll J. Long term effectiveness of ear level devices for tinnitus. Otolaryngol - Head Neck Surg 2006;134(1):132 7.

[61] Hobson J, Chisholm E, Refaie AE. Sound therapy (masking) in the management of tinnitus in adults. Cochrane Database System Rev 2010;12:1 24.

[62] Anwar MN, Oakes MP. Data mining of audiology patient records: factors influencing the choice of hearing aid type. BMC Med Inform Decision Making 2012;12.

[63] Anwar MN, Oakes MP, McGarry K. Chi squared Yule's Q and likeli hood ratios in tabular audiology data. In: Electrical engineering and applied computing. Netherlands: Springer; 2011. p. 36576.

[64] Fukuda S, Miyashita T, Inamoto R, Mori N. Tinnitus retraining therapy using portable music players. Auris Nasus Larynx 2011;38(6):692 6.

[65] Erlandsson S. Psychological profiles of tinnitus in patients. In: Tinnitus handbook. San Diego, CA: Singular Publishing Group; 2000. p. 2558.

[66] Searchfield G. A commentary on the complexity of tinnitus manage ment: clinical guidelines provide a path through the fog. Eval Health Prof 2011;34(4):421 8.

[67] Vallés Varela H, Royo López J, Carmen Sampériz L, Sebastián Cortés JM, Alfonso Collado I. El implante coclear como tratamiento del acúfeno. Acta Otorrinolaringol Española 2013;64:253 7.

[68] Caccamo S, Voloshchenko A, Dankyi NY. The importance of creating a social business to produce low cost hearing aids. Disabil Rehabil Assist Technol 2014;9(5):368 73.

[69] Seelman KD, Werner R. Technology transfer of hearing aids to low and middle income countries: policy and market factors. Disabil Rehabil Assist Technol 2014;9(5):399 407.

[70] Israsena P, Isaradisaikul S, Noymai A, Boonyanukul S, Hemakom A, Chinnarat $\mathrm{C}$, et al. Developing an appropriate digital hearing aid for low resource countries: a case study. Scientific World J 2013;2013:1 8 . 